\title{
A|P|l $\begin{aligned} & \text { The Journal of } \\ & \text { Chemical Physics }\end{aligned}$
}

\section{X-ray emission spectroscopy of bulk liquid water in "no-man's land"}

Jonas A. Sellberg, Trevor A. McQueen, Hartawan Laksmono, Simon Schreck, Martin Beye, Daniel P.

DePonte, Brian Kennedy, Dennis Nordlund, Raymond G. Sierra, Daniel Schlesinger, Takashi Tokushima, lurii Zhovtobriukh, Sebastian Eckert, Vegard H. Segtnan, Hirohito Ogasawara, Katharina Kubicek, Simone Techert, Uwe Bergmann, Georgi L. Dakovski, William F. Schlotter, Yoshihisa Harada, Michael J. Bogan, Philippe Wernet, Alexander Föhlisch, Lars G. M. Pettersson, and Anders Nilsson

Citation: The Journal of Chemical Physics 142, 044505 (2015); doi: 10.1063/1.4905603

View online: http://dx.doi.org/10.1063/1.4905603

View Table of Contents: http://scitation.aip.org/content/aip/journal/jcp/142/4?ver=pdfcov

Published by the AIP Publishing

\section{Articles you may be interested in}

An energy dispersive $\mathrm{x}$-ray scattering and molecular dynamics study of liquid dimethyl carbonate

J. Chem. Phys. 131, 244503 (2009); 10.1063/1.3273847

X-ray scattering intensities of water at extreme pressure and temperature

J. Chem. Phys. 126, 134505 (2007); 10.1063/1.2712441

Intermolecular association in liquid $\mathrm{N}$-methylacetamide as studied by $\mathrm{x}$-ray scattering

J. Chem. Phys. 121, 6380 (2004); 10.1063/1.1776554

$\mathrm{H}$-bonding in liquid acetamide as studied by $\mathrm{x}$-ray scattering

J. Chem. Phys. 110, 10487 (1999); 10.1063/1.478978

Static structure factor and electron correlation effects studied by inelastic $x$-ray scattering spectroscopy

J. Chem. Phys. 108, 4545 (1998); 10.1063/1.475865

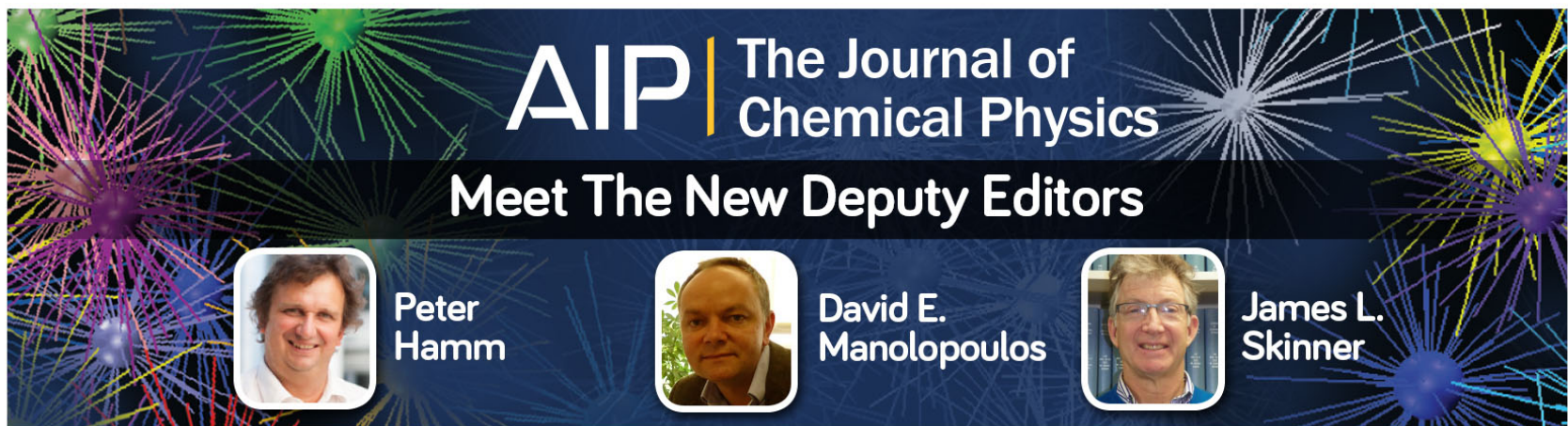




\title{
X-ray emission spectroscopy of bulk liquid water in "no-man's land"
}

\author{
Jonas A. Sellberg, ${ }^{1,2}$ Trevor A. McQueen, ${ }^{1,3}$ Hartawan Laksmono, ${ }^{4}$ Simon Schreck,, 6 \\ Martin Beye,${ }^{5}$ Daniel P. DePonte, ${ }^{7,8}$ Brian Kennedy, ${ }^{9}$ Dennis Nordlund, ${ }^{10}$ \\ Raymond G. Sierra, ${ }^{4}$ Daniel Schlesinger, ${ }^{2}$ Takashi Tokushima, ${ }^{11}$ lurii Zhovtobriukh, ${ }^{2}$ \\ Sebastian Eckert, ${ }^{5}$ Vegard H. Segtnan, ${ }^{1,12}$ Hirohito Ogasawara, ${ }^{10}$ Katharina Kubicek, ${ }^{13,14}$ \\ Simone Techert, ${ }^{14,15}$ Uwe Bergmann, ${ }^{8}$ Georgi L. Dakovski, ${ }^{8}$ William F. Schlotter, ${ }^{8}$ \\ Yoshihisa Harada, ${ }^{16,17}$ Michael J. Bogan, ${ }^{4}$ Philippe Wernet, ${ }^{5}$ Alexander Föhlisch, ${ }^{5,6}$ \\ Lars G. M. Pettersson, ${ }^{2}$ and Anders Nilsson ${ }^{1,2,10}$ \\ ${ }^{1}$ SUNCAT Center for Interface Science and Catalysis, SLAC National Accelerator Laboratory, \\ 2575 Sand Hill Road, Menlo Park, California 94025, USA \\ ${ }^{2}$ Department of Physics, AlbaNova University Center, Stockholm University, S-106 91 Stockholm, Sweden \\ ${ }^{3}$ Department of Chemistry, Stanford University, Stanford, California 94305, USA \\ ${ }^{4}$ PULSE Institute, SLAC National Accelerator Laboratory, 2575 Sand Hill Road, Menlo Park, \\ California 94025, USA \\ ${ }^{5}$ Institute for Methods and Instrumentation for Synchrotron Radiation Research, Helmholtz-Zentrum Berlin \\ für Materialien und Energie GmbH, Albert-Einstein-Straße 15, 12489 Berlin, Germany \\ ${ }^{6}$ Institut für Physik und Astronomie, Universität Potsdam, Karl-Liebknecht-Straße 24-25, 14476 Potsdam, \\ Germany \\ ${ }^{7}$ Center for Free-Electron Laser Science, DESY, Notkestraße 85, 22607 Hamburg, Germany \\ ${ }^{8}$ Linac Coherent Light Source, SLAC National Accelerator Laboratory, 2575 Sand Hill Road, Menlo Park, \\ California 94025, USA \\ ${ }^{9}$ MAX-lab, P.O. Box 118, 22100 Lund, Sweden \\ ${ }^{10}$ Stanford Synchrotron Radiation Lightsource, SLAC National Accelerator Laboratory, P.O. Box 20450, \\ Stanford, California 94309, USA \\ ${ }^{11}$ RIKEN SPring-8 Center, 1-1-1 Kouto, Sayo-cho, Hyogo 679-5148, Japan \\ ${ }^{12}$ Nofima AS, N-1430 As, Norway \\ ${ }^{13}$ Photon Science, DESY, Notkestraße 85, 22607 Hamburg, Germany \\ ${ }^{14}$ IFG Structural Dynamics of (Bio)chemical Systems, Max Planck Institute for Biophysical Chemistry, \\ Am Faßberg 11, 37070 Göttingen, Germany \\ ${ }^{15}$ Advanced Study Group of the MPG, CFEL, Notkestraße 85, 22853 Hamburg, Germany \\ ${ }^{16}$ Institute for Solid State Physics (ISSP), University of Tokyo, Kashiwanoha, Kashiwa, Chiba 277-8581, Japan \\ ${ }^{17}$ Synchrotron Radiation Research Organization, University of Tokyo, Sayo-cho, Sayo, Hyogo 679-5198, Japan
}

(Received 29 August 2014; accepted 10 December 2014; published online 28 January 2015)

\begin{abstract}
The structure of bulk liquid water was recently probed by $\mathrm{x}$-ray scattering below the temperature limit of homogeneous nucleation $\left(T_{H}\right)$ of $\sim 232 \mathrm{~K}$ [J. A. Sellberg et al., Nature 510, 381-384 (2014)]. Here, we utilize a similar approach to study the structure of bulk liquid water below $T_{H}$ using oxygen $\mathrm{K}$-edge $\mathrm{x}$-ray emission spectroscopy (XES). Based on previous XES experiments [T. Tokushima et al., Chem. Phys. Lett. 460, 387-400 (2008)] at higher temperatures, we expected the ratio of the $1 b_{1}{ }^{\prime}$ and $1 b_{1}{ }^{\prime \prime}$ peaks associated with the lone-pair orbital in water to change strongly upon deep supercooling as the coordination of the hydrogen (H-) bonds becomes tetrahedral. In contrast, we observed only minor changes in the lone-pair spectral region, challenging an interpretation in terms of two interconverting species. A number of alternative hypotheses to explain the results are put forward and discussed. Although the spectra can be explained by various contributions from these hypotheses, we here emphasize the interpretation that the line shape of each component changes dramatically when approaching lower temperatures, where, in particular, the peak assigned to the proposed disordered component would become more symmetrical as vibrational interference becomes more important. @ 2015 AIP Publishing LLC. [http://dx.doi.org/10.1063/1.4905603]
\end{abstract}

\section{INTRODUCTION}

Water has been studied intensively for hundreds of years but is still far from fully understood. Knowledge about the $\mathrm{H}$-bond network in water is essential for understanding its numerous unusual chemical and physical properties. For example, water can exist in the liquid state far below its melting temperature (supercooled water) where its thermodynamic response functions, such as coefficient of thermal expansion $\left(\alpha_{P}\right)$, isothermal compressibility $\left(\kappa_{T}\right)$, isobaric heat capacity $\left(C_{P}\right)$, and correlation length $(\xi)$, appear to diverge at a difficult to reach temperature of $228 \mathrm{~K}^{1-3}$ Water crystallization occurs very rapidly below the homogeneous nucleation temperature $\left(T_{H}\right)$ of $\sim 232 \mathrm{~K},{ }^{4}$ which has prevented measurements of the liquid phase and led to a "no-man's land" devoid of experimental results. The anomalous behavior of water 
becomes important already at ambient conditions giving a maximum density at $277 \mathrm{~K}$, and a minimum in $C_{P}$ and $\kappa_{T}$ at $308 \mathrm{~K}$ and $319 \mathrm{~K}$, respectively.

The apparent divergence of $C_{P}$ and $\kappa_{T}$ upon supercooling has led to several proposed scenarios involving fluctuations between local high-density liquid (HDL) and low-density liquid (LDL) configurations. These fluctuations could, upon deeper supercooling, lead either to a liquid-liquid transition (LLT) with a liquid-liquid critical point (LLCP) at positive pressure $^{5}$ or a continuous transformation without discontinuity, called the singularity-free (SF) model. ${ }^{6}$ In the critical point-free (CPF) model, the LLCP would instead occur at negative pressure ${ }^{7}$ (outside the physically relevant part of the phase diagram), but there is also a recent suggestion that only a liquid-solid transition exists, ${ }^{8}$ which is currently under debate. ${ }^{9-14}$ Alternatively, a thermodynamic stability limit (SL) of the liquid state has been suggested ${ }^{15}$ and that ice crystallization occurs on a timescale faster than liquid equilibration in "no-man's land."16 These scenarios do not agree, and to date convincing experimental data have been lacking. Recently, a new technique has been demonstrated where it is possible to enter "no-man's land" through fast evaporative cooling of micron-sized water droplets and ultrafast probing using x-ray scattering from an X-ray laser. ${ }^{17}$ It was shown that water could be probed in a metastable liquid phase down to $227 \mathrm{~K}$ and that a continuous increase of structures with local tetrahedral coordination became more enhanced upon deep supercooling, which indicates an accelerated transition toward LDL.

There are several spectroscopic techniques such as vibrational spectroscopy and core-level spectroscopy that are sensitive to the hydrogen-bonding (H-bonding) environment. ${ }^{18} \mathrm{In}$ particular, recent $\mathrm{X}$-ray emission (XE) spectra show a split of the lone-pair $\left(1 b_{1}\right)$ peak in water ${ }^{19,20}$ that has been interpreted as arising from structures with distorted H-bonds $\left(1 b_{1}{ }^{\prime \prime}\right)$ and from tetrahedrally H-bonded structures $\left(1 b_{1}{ }^{\prime}\right){ }^{18,20-27}$ As the liquid temperature is raised, intensity is transferred from the low-energy $1 b_{1}{ }^{\prime}$ peak to the high-energy $1 b_{1}{ }^{\prime \prime}$ peak, but no broadening or additional features are observed. ${ }^{20,28}$ Also, selective excitations show a clear correlation between the two $1 b_{1}$ peaks and the various resonances in the $\mathrm{x}$-ray absorption spectrum of water consistent with the hypothesis of two structural motifs. ${ }^{20,28}$ An interpretation has been proposed in terms of these local structures being related to fluctuations in the liquid giving rise to local regions of HDL (distorted) and LDL (tetrahedral) character. ${ }^{18,24,28,29}$

However, the strong isotope effect in the spectra has led to other suggestions of the origin of the two $1 b_{1}$ peaks..$^{19,20}$ The first alternative interpretation suggests that the two peaks are due to intermediate or final states of ultrafast dissociation during the core-hole lifetime. ${ }^{19,30}$ The peak at low emission energy was proposed to come from $\mathrm{OH}$ species generated from water through core-hole-induced dissociation while the other was proposed to be due to intact water. ${ }^{19,30}$ The second alternative interpretation, based on theoretical simulations, proposed that the peak at high energy is of lone-pair $1 b_{1}$ symmetry, whereas the peak at low emission energy is of $3 a_{1}$ symmetry and originating from the strong dynamical response toward dissociation found in the simulations. ${ }^{31,32}$ Excitationselective, polarization-dependent measurements indicated first that there could be a difference between the two peaks in terms of symmetry, ${ }^{33}$ but later more data and better statistics demonstrated that the two lone-pair peaks are both of $b_{1}$ symmetry ruling out that the $1 b_{1}{ }^{\prime}$ peak would be of $a_{1}$ symmetry. ${ }^{27}$ Furthermore, theoretical calculations of the XE spectrum of a model water dimer including vibrationallifetime interference in a full Kramers-Heisenberg treatment show only the appearance of an asymmetrical line shape and not two distinct peaks. ${ }^{34,35}$ The asymmetry of the $b_{1}$ peak of the lighter isotope is much larger which could explain the isotope effect.

Based on the interpretation in terms of two structural motifs, we would expect a rapid rise in intensity for the $1 b_{1}{ }^{\prime}$ peak if the liquid is cooled down to temperatures within "noman's land." Here, we show that this is not the case. We will review several possible explanations for this result. Although no definite conclusion can be drawn, it is essential that these data are made available, since it is the first x-ray emission spectroscopy (XES) study of liquid water venturing into "noman's land" and since it led to results in seeming contradiction to expectation.

\section{METHODS}

\section{A. Experimental setup at the Soft X-ray Materials Science (SXR) instrument, Linac Coherent Light Source (LCLS)}

Non-resonant O K-edge XES was performed at the SXR instrument ${ }^{36}$ at the LCLS using a nominal photon energy of $550 \mathrm{eV}$ (well above the $\mathrm{O}$ K-edge absorption resonance) and a nominal pulse duration of $100 \mathrm{fs}$. These pulses, delivered at $120 \mathrm{~Hz}$ and containing between $6 \times 10^{11}$ and $12 \times 10^{11}$ photons/pulse (accounting for $10 \%$ beamline transmission from gas detectors to the sample ${ }^{37}$ ), were focused to a focal spot size of $75 \times 520 \mu \mathrm{m}^{2}$ (horizontal $\times$ vertical) at the interaction point, corresponding to a peak fluence of $0.27 \mathrm{~J} / \mathrm{cm}^{2}$, which is comparable to the threshold of $\sim 0.2 \mathrm{~J} / \mathrm{cm}^{2}$ where a non-linear fluence dependence of the $\mathrm{XE}$ yield starts to occur due to valence-hole reabsorption. ${ }^{37}$ Liquid $\mathrm{D}_{2} \mathrm{O}$ (Fischer Scientific \#AC166311000, 99.95\%; and Sigma-Aldrich \#151882, 99.9 at. \%) was delivered to the interaction region using the Liquid Jet End Station, which consists of a liquid microjet in a differentially pumped high vacuum $\left(2 \times 10^{-3}\right.$ millibars at the jet, $4 \times 10^{-7}$ millibars at the detector) and a Graze spectrometer XES $350^{38}$ mounted at $90^{\circ}$ with respect to the incident $x$-ray beam, as described in detail elsewhere. ${ }^{39}$ The liquid microjet was produced by a gas-dynamic virtual nozzle ${ }^{40}$ (GDVN) operated at 800 psi liquid pressure and 330 psi He gas pressure. The microjet underwent Rayleigh breakup ${ }^{41}$ after a few hundred microns, driven by a piezoelectric actuator at $900 \mathrm{kHz}$ (sine wave with $50 \mathrm{~V}$ amplitude), producing a singlefile train of uniform droplets with a diameter of $5 \mu \mathrm{m}$, droplet spacing of $16 \mu \mathrm{m}$, and droplet speed of $14 \mathrm{~m} / \mathrm{s}$. The droplet diameter $D_{\text {drop }}$, droplet spacing $l_{\text {drop-drop }}$, and droplet speed $v$ were estimated by mass conservation for a single-file droplet train of uniform diameter, namely, $v=Q / A_{\text {jet }}, l_{\text {drop-drop }}=v / f$, and $D_{\text {drop }}=2(3 Q / 4 \pi f)^{1 / 3}$, where $f$ is the droplet frequency determined by the piezoelectric actuator, $Q$ is the in situ 
measured volume flow rate of $3.6 \mu \mathrm{l} / \mathrm{min}$, and $A_{\text {jet }}$ is the crosssectional area of the initial liquid jet, determined to be $4.2 \mu \mathrm{m}^{2}$ at given flow rate using a scanning electron microscope. Due to the large vertical focal spot size compared to the droplet spacing, up to 34 droplets were probed simultaneously for each shot; each spectrum was averaged over up to $2 \times 10^{6}$ shots, resulting in at least 90000 detected photons. The distance between the dispenser nozzle and the interaction point, which determines the temperature of water droplets, is controlled by a 3 -axis motorized sample stage. The temperature was estimated using Knudsen theory of evaporation, ${ }^{42-44}$ which is mainly dependent on the droplet diameter, droplet speed, and the extrapolation of thermodynamic parameters into "no-man's land" (see supplementary information in Sellberg et al. ${ }^{17}$ for details). During cooling, the droplets lose up to about $13 \%$ of their mass through evaporation; the temperature within individual droplets can be considered homogeneous with differences less than $0.2 \mathrm{~K}$ between core and surface after $1 \mathrm{~ms}$ of travel time. The cooling is expected to be delayed due to the surrounding $\mathrm{He}$ gas, resulting in a temperature close to the room temperature $T_{0} \approx 293 \mathrm{~K}$ for measurements up to $0.8 \mathrm{~mm}$ away from the nozzle. Hence, the total uncertainty in temperature is estimated to be $290_{-7}^{+3} \mathrm{~K}, 226_{-1}^{+2} \mathrm{~K}$, and $222_{-3}^{+5} \mathrm{~K}$ at $0.6 \pm 0.4 \mathrm{~mm}, 24.4 \pm 0.4 \mathrm{~mm}$, and $45.5 \pm 0.3 \mathrm{~mm}$ away from the nozzle, respectively, where XE spectra were recorded. Surface tension generates a pressure on droplets which here, however, is negligible, since it corresponds to a pressure increase of $\sim 0.6$ bar.

\section{B. Data analysis at the SXR instrument, LCLS}

Emission spectra were recorded at $550 \mathrm{eV}$ incident photon energy on a CsI coated multi-channel plate (MCP) stack combined with a phosphor screen monitored by a chargecoupled device camera (Opal 1000, Adimec), read out by the LCLS data acquisition system. Data were processed by singlephoton identification. The photon positions were characterized by the center of mass of the fluorescent signal and translated to energy scale by projection onto the $x$-axis of the detector after a quadratic curvature correction. The absolute energy was calibrated by shifting the $1 b_{1}{ }^{\prime \prime}$ and $1 b_{2}$ peaks of the spectrum recorded at $\sim 290 \mathrm{~K}$ to their previously determined positions ${ }^{28}$ of $526.78 \mathrm{eV}$ and $521.10 \mathrm{eV}$, respectively; the corresponding $\mathrm{XE}$ spectra had an energy binning of $\sim 75 \mathrm{meV}$. All XE spectra were background subtracted with an 8th order polynomial and area normalized between $500 \mathrm{eV}$ and $550 \mathrm{eV}$.

\section{Experimental setup at BL17SU and BL07LSU, SPring-8}

Temperature-dependent O K-edge XES was performed at BL17SU at SPring-8, Japan, details of which have been described elsewhere. ${ }^{45}$ The fluence dependence experiment was performed at BL07LSU at SPring-8, Japan. The liquid flow-through cell system used was basically the same as used at $\mathrm{BL} 17 \mathrm{SU},{ }^{26}$ whereas the $\mathrm{XE}$ spectrometer has higher total energy resolution ${ }^{46}(\mathrm{E} / \Delta \mathrm{E} \sim 5000)$ than the one at BL17SU due to its long optical path length as well as coma-free mode operation. ${ }^{47}$ The incident photon flux was varied by tuning the front end slit placed at the upper stream of the monochromator, which only changes the fluence but affects neither the energy resolution nor the spot size at the sample position. Using this setup, we can thus precisely study the fluence dependence of the $\mathrm{O}$ K-edge XE spectra of liquid $\mathrm{H}_{2} \mathrm{O}$. In contrast to the free-electron laser measurements described in Sec. II A, the synchrotron measurements at SPring-8 were performed with picosecond x-ray pulses $(\sim 50 \mathrm{ps})$ at much higher repetition rates ${ }^{48}(\sim 500 \mathrm{MHz})$, resulting in that more than one pulse probe the same volume and that much lower fluences are obtained than at the free-electron laser. Hence, the fluence measurements are very different from the previous study by Schreck et $a l^{37}$ using LCLS to probe valence-hole reabsorption.

\section{Simulated XE spectra}

The calculations were performed using density functional theory for cluster models containing 32 molecules. Ten structures were extracted as a sampling of the vdW-DF2 $a b$ initio molecular dynamics NVE simulation of 64 water molecules performed by Møgelhøj et al. ${ }^{49}$ The central water was computed as either intact or as one of the fragments $(\mathrm{O}$, $\mathrm{O}^{+}, \mathrm{OH}, \mathrm{OH}^{+}$). Spectra were calculated using the ground state orbitals ${ }^{50}$ and shifted such that the $1 b_{1}{ }^{\prime \prime}$ peak computed for $\mathrm{H}_{2} \mathrm{O}$ coincides with the experimental position; spectra for the fragments were shifted by the same amount assuming that the neglected relaxation effects are similar in all cases. Emission energies and transition probabilities were computed with orbital-based density functional theory with the deMon2k code $^{51}$ and the revised Perdew, Burke, and Ernzerhof ${ }^{52}$ (revPBE) gradient-corrected functional. The IGLO-III basis set of Kutzelnigg et al. ${ }^{53}$ was used to represent the oxygen of the core-excited molecule. In the other oxygen, the $1 s$ core electrons were replaced by an effective core potential ${ }^{54}$ and the remaining electrons were described using $4 s 4 p$ basis functions contracted to $2 s 2 p$. All hydrogens were described using $5 s 2 p$ functions contracted to $2 s 2 p$.

\section{RESULTS}

Fig. 1 shows XE spectra measured at LCLS at nonresonant conditions $(550 \mathrm{eV})$ on the water droplets at three different temperatures corresponding to $\sim 290 \mathrm{~K}, 226_{-1}^{+2} \mathrm{~K}$, and $222_{-3}^{+5} \mathrm{~K}$. We notice quite small changes in the relative peak heights of the low-energy $1 b_{1}{ }^{\prime}$ and high-energy $1 b_{1}{ }^{\prime \prime}$ peaks, but the $1 b_{1}{ }^{\prime \prime}$ peak width narrows with decreasing temperature, resulting in a shift of the center of mass of the peak from 526.74 $\mathrm{eV}$ at $\sim 290 \mathrm{~K}$ to $526.66 \mathrm{eV}$ and $526.63 \mathrm{eV}$ at $226_{-1}^{+2} \mathrm{~K}$ and $222_{-3}^{+5} \mathrm{~K}$, respectively. Because the XE spectrum of crystalline ice ${ }^{55}$ only shows a prominent $1 b_{1}{ }^{\prime}$ peak with the high-energy $1 b_{1}{ }^{\prime \prime}$ peak reduced to a weak shoulder, we may exclude that a significant volume fraction of the illuminated droplets would have crystallized prior to detection upon supercooling the liquid. We note that the two peaks are less well-resolved in comparison to previous measurements, which can be attributed to different energy resolutions. Fig. 2 shows that the LCLS data at $\sim 290 \mathrm{~K}$ can be fitted with relative intensities between the 


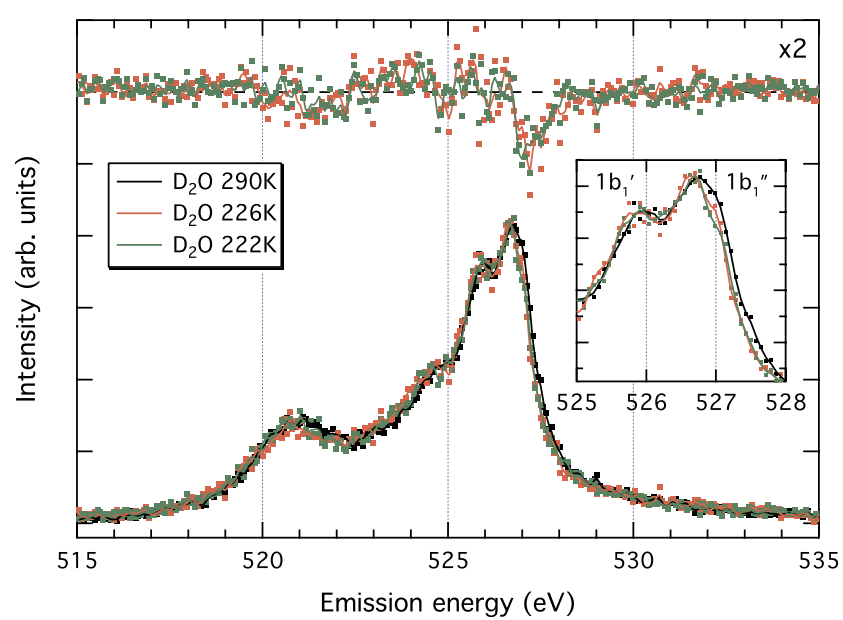

FIG. 1. O K-edge XE spectra of liquid $\mathrm{D}_{2} \mathrm{O}$ measured at $\sim 290 \mathrm{~K}$ (black curve), $226 \mathrm{~K}$ (red curve), and $222 \mathrm{~K}$ (green curve). The XE spectra were recorded at LCLS using an incident photon energy of $550 \mathrm{eV}$, a nominal pulse length of $100 \mathrm{fs}$, and a beam focus of $75 \times 520 \mu \mathrm{m}^{2}$ (horizontal $\times$ vertical) at the interaction point, corresponding to a peak fluence of $0.27 \mathrm{~J} / \mathrm{cm}^{2}$. The top shows the difference spectra (magnified by a factor of 2) with respect to the XE spectrum of $\mathrm{D}_{2} \mathrm{O}$ at $290 \mathrm{~K}$. The inset shows an enlarged view of the lone-pair $\left(1 b_{1}\right)$ region of the XE spectra. All XE spectra were background subtracted with an 8th order polynomial and area normalized between 500 and $550 \mathrm{eV}$.

two components similar to high-resolution data measured at BL17SU at SPring-8 at $300 \mathrm{~K}$ if one accounts for the different instrumental resolutions and an additional background in the LCLS data from diffuse scattering from the grating due to imperfections, most likely originating from contamination.

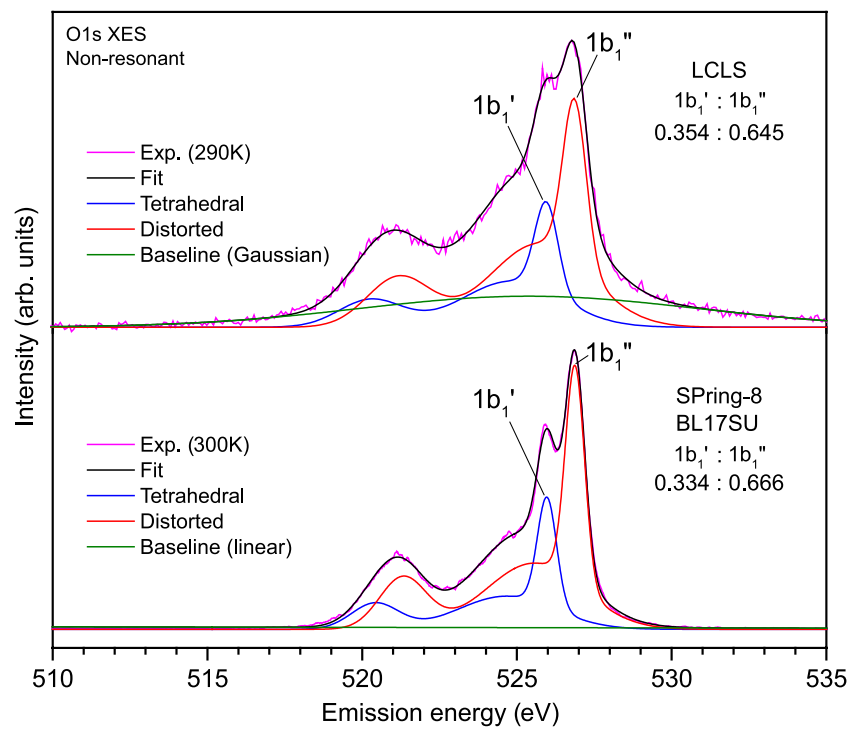

FIG. 2. Gaussian peak-fit analysis of $\mathrm{O}$ K-edge XE spectra of liquid $\mathrm{D}_{2} \mathrm{O}$ measured at LCLS at $\sim 290 \mathrm{~K}$ (top) and SPring8 at $300 \mathrm{~K}$ (bottom). The spectra are decomposed into two sets of three Gaussians, corresponding to the molecular orbitals $1 b_{2}, 3 a_{1}$, and $1 b_{1}$. Each set represents a molecular species and is separated by an absolute energy shift, representing a core-level shift between the two hypothesized species, and an amplitude difference, representing the relative population of each species. The high-energy set (red curve) corresponds to species with distorted H-bonds, whereas the low-energy set (blue curve) corresponds to tetrahedrally H-bonded species. For the LCLS data, a Gaussian background (green curve) is necessary to describe the diffuse background scattering from the grating due to imperfections most likely originating from contamination.
Based on that the spectra at $\sim 290 \mathrm{~K}$ measured at LCLS and at $300 \mathrm{~K}$ measured at SPring- 8 can be fitted with similar populations of species related to distorted and tetrahedral $\mathrm{H}$ bonds, we can decompose the spectra into relative populations as a function of temperature using the LCLS data at lower temperatures while using the SPring- 8 data ${ }^{20,28}$ at higher temperatures. For this decomposition, we assume that the line shapes of the two components are independent of temperature. Fig. 3 shows the temperature dependence of the relative populations of the $1 b_{1}{ }^{\prime}$ and $1 b_{1}{ }^{\prime \prime}$ components as obtained under this assumption. It is clear that the fraction of distorted $\mathrm{H}$-bonds is decreasing and the fraction of tetrahedral $\mathrm{H}$ bonds is increasing with decreasing temperature, consistent with previous findings. ${ }^{20,28}$ Furthermore, we also observe the change in the high-energy $1 b_{1}{ }^{\prime \prime}$ peak position toward lower emission energy (Fig. 1) to be consistent with the previous studies. However, there is a major discrepancy as to what we would expect in terms of intensity of the low-energy $1 b_{1}$ " peak in "no-man's land" where the liquid is expected to become LDL-like with tetrahedral H-bonds. Analysis of inherent structures from the TIP4P/2005 force field shows that at $230 \mathrm{~K}$ the LDL and HDL populations should reach a 1:1 ratio and at temperatures below $230 \mathrm{~K}$ the LDL population should dominate..$^{24,56}$ This is supported by experiments in "no-man's land" where it was found that the tetrahedrality in terms of the peak height of the 2nd shell in the O-O pair correlation function becomes exceptionally strong and mimics low-density amorphous (LDA) ice. ${ }^{17}$ The experimental temperature-dependent trend shows an even stronger increase in tetrahedrality than what is obtained from the TIP4P/2005 simulations. ${ }^{17}$ We would therefore expect that at $226 \mathrm{~K}$ a crossing between the $1 b_{1}{ }^{\prime}$ and $1 b_{1}{ }^{\prime \prime}$ populations would have occurred with the low-energy $1 b_{1}{ }^{\prime}$

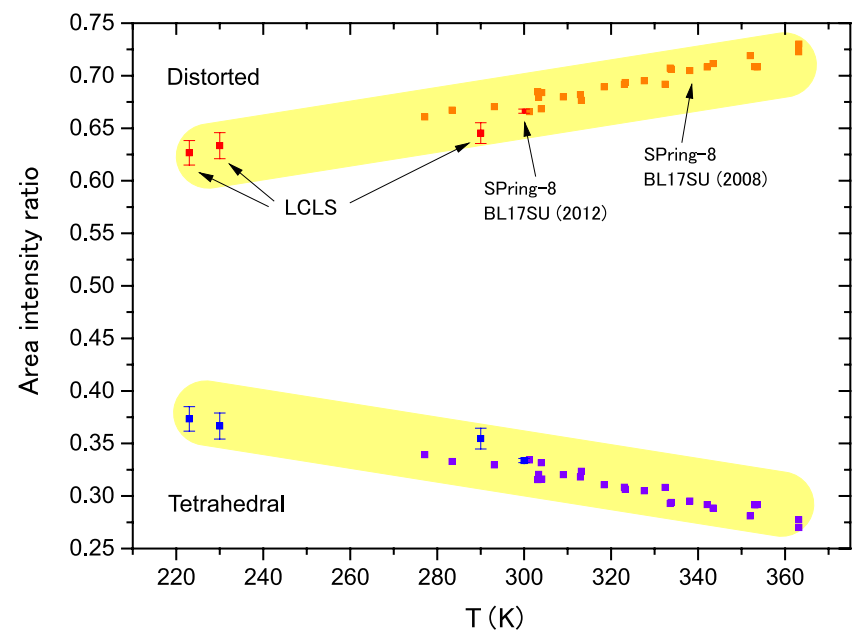

FIG. 3. Population analysis of tetrahedrally H-bonded species (blue and purple markers) and species with distorted H-bonds (red and orange markers) deduced from Gaussian peak-fit analysis of O K-edge XE spectra of liquid $\mathrm{D}_{2} \mathrm{O}$ measured at LCLS in 2012, SPring-8 in 2012, and SPring- 8 in $2008^{28}$ as marked in the figure. The vertical error bars for spectra measured at LCLS in 2012 and at SPring- 8 in 2012 are estimated by the standard errors for the intensity area of each species, corresponding to one standard deviation in the confidence interval for the amplitude difference in the peak-fit analysis (Fig. 2). Within the model assumed, the tetrahedral species is a minority at all temperatures, which is in disagreement with recent $\mathrm{x}$-ray scattering measurements. ${ }^{17}$ 
peak (interpreted as corresponding to local regions of LDL) becoming dominant. Clearly, this is not observed in Fig. 3.

\section{DISCUSSION}

The lack of strong temperature dependence in the peak heights of the $1 b_{1}{ }^{\prime}$ and $1 b_{1}{ }^{\prime \prime}$ peaks opens questions regarding our detailed understanding of the XE process and its connection to the structure of the liquid. The XE energy corresponds to the difference in binding energy of the $1 b_{1}$ and $\mathrm{O} 1 s$ orbitals as could also be measured through photoelectron spectroscopy. We note first that the valence photoelectron spectrum of water only shows one peak in the lone-pair region and that this is too sharp to support two contributions at the separation observed in XES. ${ }^{57-59}$ Core-level photoelectron spectroscopy of liquid water shows only one O1s peak as well, but in this case the O1s peak is broad enough to support two unresolved features within the separation between the two $1 b_{1}$ peaks in XES.$^{60}$ Here, we will discuss various hypotheses that can explain the current data. We are thereby revisiting an old discussion, ${ }^{25}$ but with a focus on that the temperature dependence is somewhat unexpected.

First, could the dissociative mechanism better explain the appearance of the two peaks? $?^{19,31,32}$ This has recently been extensively reviewed ${ }^{25}$ and excluded based both on highlevel theoretical simulations of a model water dimer ${ }^{34,35}$ and polarization-dependent measurements. ${ }^{27}$ It was shown that, although the potential energy surface in the core-excited state has its minimum at the accepting oxygen, the proton becomes delocalized between the two water molecules and the resulting $\mathrm{O}-\mathrm{H}$ stretch has to be described as a superposition of several excited vibrational states, giving rise to important interference effects when the appropriate Kramers-Heisenberg formalism is applied. Indeed, the computed XE spectrum of the water dimer does not exhibit two distinct peaks but rather an asymmetrical $1 b_{1}$ line shape that can also explain the isotope effect. ${ }^{25,34,35}$ Furthermore, the difference in binding energy between $1 b_{1}$ and $\mathrm{O} 1 s$ in photoelectron spectroscopy is nearly identical for $\mathrm{OH}$ and water, ${ }^{26}$ thus not giving an extra feature in the $1 b_{1}$ region and, in addition, we would expect to see a distinct feature at $523 \mathrm{eV}$ characteristic of a well-defined $\mathrm{OH}$ species. ${ }^{25}$ A dissociative mechanism involving $\mathrm{OH}$ and giving a sharp spectral feature in the lone-pair spectrum, but not at $523 \mathrm{eV}$, thus cannot account for the dependence of the two peaks on temperature, ${ }^{28}$ polarization, ${ }^{27}$ and excitation energy. ${ }^{20,25,27,28}$

A second possibility is that the two peaks observed in XES reflect an additional and different bimodal distribution than that given by distorted and tetrahedral H-bonds which then could have temperature dependence different from that expected for HDL and LDL from molecular dynamics simulations. This seems highly unlikely, however, and there is currently no suggestion from simulations or theoretical considerations of any additional division of structures into classes with the observed weak temperature dependence and which would give rise to the two peaks in XES but be distinct from the suggested species.

A third explanation could be that the split is due to a ground state electronic structure effect in the liquid which

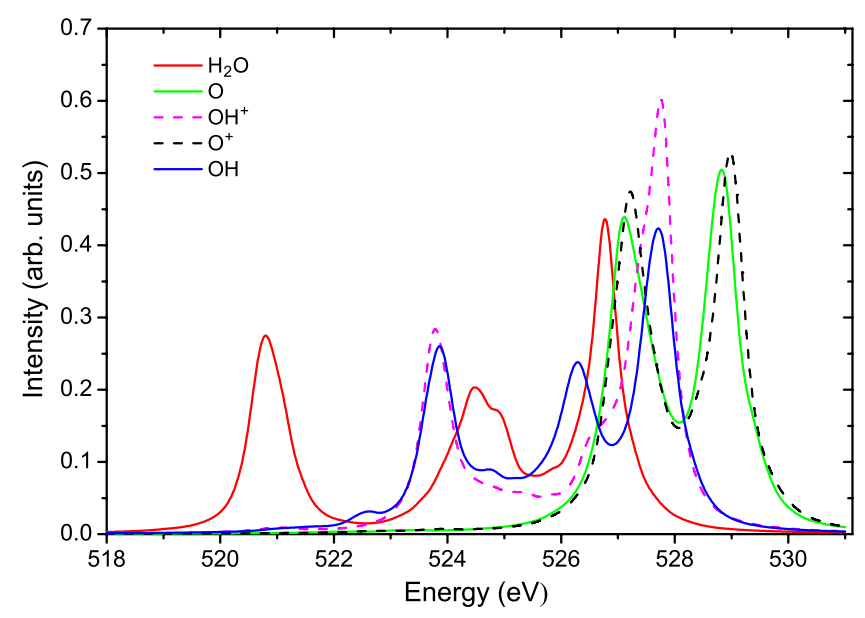

FIG. 4. Computed XE spectra for $\mathrm{H}_{2} \mathrm{O}$ (red solid curve) and neutral (solid curves) and positive (dashed curves) fragments of $\mathrm{OH}$ and atomic $\mathrm{O}$ in a sampling of 32 molecules water clusters (Methods). The same shift has been applied to all spectra such that the $1 b_{1}$ peak position of $\mathrm{H}_{2} \mathrm{O}$ coincides with the experimental $1 b_{1}{ }^{\prime \prime}$ peak position. Contributions from the fragments appear in the vicinity of the high-energy $1 b_{1}{ }^{\prime \prime}$ peak but do not contribute to the $1 b_{1}{ }^{\prime}$ peak at lower emission energy.

depends on the local environment where the $1 b_{1}$ peak is split due to band structure effects as for the $3 a_{1}$ in ice. ${ }^{57,61}$ The argument against such an interpretation is that the valence photoelectron spectrum of liquid water should then also show two such peaks in the lone-pair region. ${ }^{57,61} \mathrm{In}$ particular, measurements at relatively low photon energy, where the photoionization cross section is dominated by the $\mathrm{O} 2 p$ contribution, ${ }^{57,61}$ should give spectra similar to XES which is also completely derived from the $\mathrm{O} 2 p$ character through the dipole selection rule for O K-edge emission. This, however, is not observed.

A 4th hypothesis is that there could be radical species building up in the liquid, not from ultrafast dissociation, but from the Auger decay cascade. ${ }^{62}$ These could include various fragmented water species, such as the $\mathrm{OH}$ radical, that could contribute to the $1 b_{1}{ }^{\prime}$ peak. This is contradicted by theoretical calculations of the $1 b_{1}$ to $\mathrm{O} 1 s$ emission energy for $\mathrm{OH}, \mathrm{OH}^{+}$, $\mathrm{O}$, and $\mathrm{O}^{+}$as fragments, which all lead to a contribution at the $1 b_{1}{ }^{\prime \prime}$ position of intact water or at higher energy (Fig. 4). The theoretical results are further supported by experiments shown in Fig. 5 where high-resolution spectra of room temperature water, in this case $\mathrm{H}_{2} \mathrm{O}$, were measured at different incident fluence at BL07LSU at SPring-8 to see if the $1 b_{1}{ }^{\prime}$ could be related to some fragmentation processes; since more fragments would be expected at higher fluence, the $1 b_{1}{ }^{\prime}$ peak would be expected to be enhanced at higher fluence. In agreement with the calculations, no specific additional intensity grows in the $1 b_{1}{ }^{\prime}$ region with increasing fluence, indicating that this feature is not related to $\mathrm{OH}, \mathrm{OH}^{+}, \mathrm{O}$, or $\mathrm{O}^{+}$fragments. However, there is a small and almost negligible conversion of some $1 b_{1}{ }^{\prime}$ to $1 b_{1}{ }^{\prime \prime}$ intensity with increasing fluence that will be discussed below. Although the currently tested radical species are the most probable ${ }^{62}$ we cannot definitely rule out that there could exist other species not tested here that could give rise to spectral intensity at lower energies. We note that non-linear effects in terms of reabsorption can affect the spectra but only 


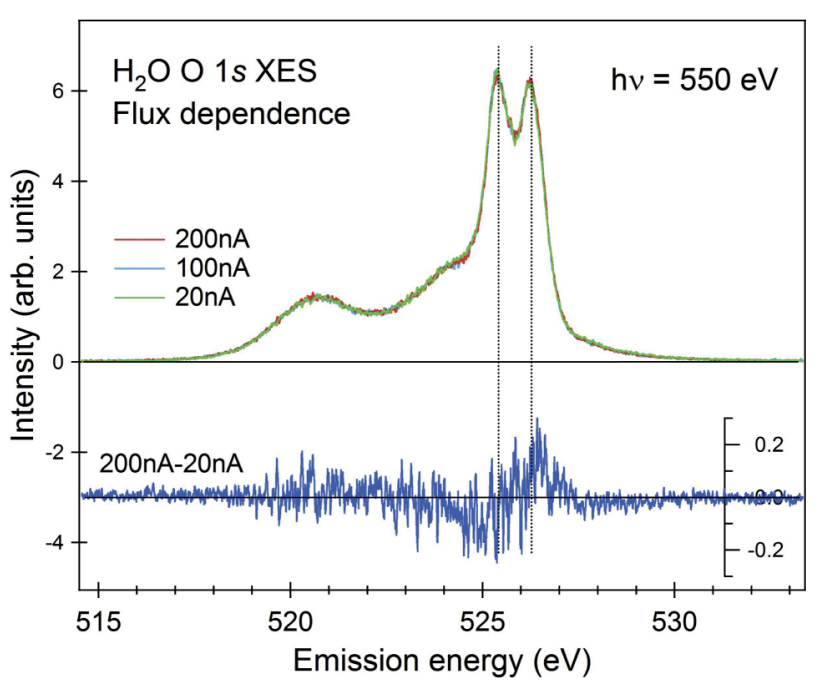

FIG. 5. Flux-dependent $\mathrm{O}$ K-edge XE spectra of liquid $\mathrm{H}_{2} \mathrm{O}$ measured at BL07LSU at SPring-8. Incident beam intensity was measured by a drain current of the post-focusing mirror placed $150 \mathrm{~mm}$ upstream of the sample position, and tuned from minimum ( $20 \mathrm{nA}$, green curve) to maximum (200 nA, red curve). The intensity was normalized by the displayed area intensity. The lower blue curve is the difference spectrum between the normalized 200 nA and 20 nA spectra.

at significantly higher fluence than the peak fluence in the present work. ${ }^{37}$

A 5th hypothesis is that there is beam-induced transformation, not in terms of dissociation but in terms of local structural environment. This has been suggested in a recent study of the $\mathrm{x}$-ray absorption spectra of cubic and hexagonal ice in order to explain the variation of the pre-edge intensity in various samples and using different measurement techniques. ${ }^{63}$ It was proposed that even if the ice is prepared as a pure hexagonal ice phase, the interaction with the beam may transform portions of the ice from hexagonal to high-density amorphous (HDA) ice. It is known that the enthalpy difference between ice $I_{h}$ and HDA ice is $0.58 \pm 0.1 \mathrm{kcal} / \mathrm{mol}^{64}$ at 1 atm while the absorption of a photon at $550 \mathrm{eV}$ corresponds to 12680 $\mathrm{kcal} / \mathrm{mol}$, which is 4 orders of magnitude higher and thus more than sufficient to convert ice $I_{h}$ into HDA ice if the energy is not efficiently transferred away from the excited molecule. We postulate that the absorbed energy will dissipate slowly through the solid in the case of ice, creating many sites where significant energy is dumped and causing a local pressure rise that converts a fraction of the volume into HDA ice. Since the low temperature hinders thermal motions, these molecules are kinetically hindered to convert back to low-density hexagonal ice.

In the liquid state, at ambient temperatures, the large thermal motion and ultrafast dynamics of breaking and forming $\mathrm{H}$ bonds on a picosecond timescale ${ }^{65}$ allow for a beam-induced structural defect to convert back nearly instantaneously and no beam-induced structural transformation will be seen. This can account for the almost negligible intensity redistribution at room temperature in connection to Fig. 5. However, as water is supercooled below $T_{H}$, the relaxation time in the liquid will increase significantly. It has been shown that the structural relaxation time at $220 \mathrm{~K}$ in simulations of liquid water using the TIP4P/2005 water model is $\sim 10 \mathrm{~ns} .{ }^{66}$ Under a certain flux that transforms the liquid and if the rate to transform back to the structures at equilibrium is slow due to long relaxation time there could build up a steady-state concentration in deeply supercooled water of distorted $\mathrm{H}$-bonds through this deposited energy resulting in converting some of the $1 b_{1}{ }^{\prime}$ to $1 b_{1}{ }^{\prime \prime}$ intensity. The effect would become more prominent the colder the liquid becomes through the increasing relaxation time. The challenge to this hypothesis is that the $\mathrm{x}$-ray pulse from LCLS is only around $100 \mathrm{fs}$ in duration and the question is if the liquid would have time to undergo any transformation within this time period. A vibrational period in the H-bond translational mode is around $200 \mathrm{fs},{ }^{67,68}$ which is relatively slow in comparison to the pulse length. However, regarding the deposited energy as equivalent to a local heating of the liquid it is most likely the librational mode that will mediate a structural transformation, where the water molecule will rotate out to avoid directional H-bonds. When comparing HDA ice to LDA ice, it is mostly bending of H-bonds that occurs $^{69}$ and we anticipate similar changes in a conversion of tetrahedrally $\mathrm{H}$-bonded structures to local structures with distorted $\mathrm{H}$-bonds at low temperatures. The vibrational period of the librational mode is of the order of $50 \mathrm{fs}$ and a fractional period of motion could occur on a few femtoseconds to tens of femtoseconds timescale. This could result in a high rate of structural transformation, since the intensity during the LCLS pulse is very high, allowing for many excited molecular centers. Note however, that even if such a conversion would occur in soft x-ray experiments using free-electron lasers, we do not expect this effect to be significant for the recently performed hard x-ray scattering experiment at LCLS, ${ }^{17}$ since at $9.4 \mathrm{keV}$ the absorption cross section is lowered by several orders of magnitude in comparison to just above the $\mathrm{O} \mathrm{K}$ edge.

Finally, a 6th hypothesis is that a modified fitting scheme of the two components is required, where also the line shape depends on temperature. It is most likely that the local structures with distorted and tetrahedral H-bonds will undergo changes with temperature. This is seen through the high emission energy $1 b_{1}{ }^{\prime \prime}$ peak position changing with temperature ${ }^{20,28}$ similarly to what is also seen in the XAS temperature-dependent spectra. ${ }^{28,70}$ We could view the two structural motifs as two different ensembles of molecules so that the line shape of the two peaks could depend on the temperature and not only on the distribution of species within each subensemble. This could occur through the local environment affecting the vibrational lifetime interference causing a change in the degree of asymmetry in the line shape. Let us first demonstrate that this is possible by simply changing the line width of the $1 b_{1}{ }^{\prime}$ and $1 b_{1}{ }^{\prime \prime}$ components in the $222 \mathrm{~K}$ spectrum as shown in Fig. 6. Since the spectral resolution in the LCLS spectra is lower than in the SPring-8 spectra, the minimum between the $1 b_{1}{ }^{\prime}$ and $1 b_{1}{ }^{\prime \prime}$ peaks is not well resolved in the LCLS spectra. This, together with the lower signal-tonoise ratio, allows for a wide range of spectral component widths to be consistent with the data and opens up a possibility that these can indeed undergo a large change with temperature, in particular in the deeply supercooled region; note that the higher resolution and better statistics of the SPring- 8 spectra puts stricter limits on this range. Fig. 6 demonstrates that nearly any ratio between the two components can be achieved 


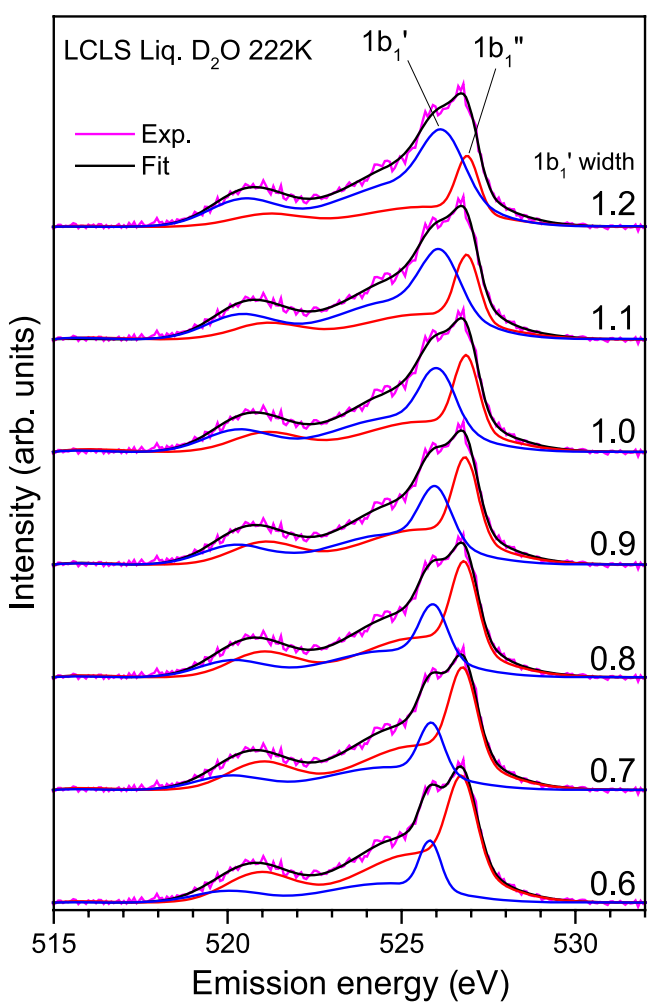

FIG. 6. Peak-fit analysis of the XE spectrum of liquid $\mathrm{D}_{2} \mathrm{O}$ at $222 \mathrm{~K}$ measured at LCLS as a function of different line widths of the $1 b_{1}{ }^{\prime}$ (given to the right of each spectrum) and $1 b_{1}{ }^{\prime \prime}$ components. The $1 b_{1}{ }^{\prime} / 1 b_{1}{ }^{\prime \prime}$ intensity ratio as well as the $1 b_{1}{ }^{\prime} / 1 b_{1}{ }^{\prime \prime}$ line width ratio increases monotonically with increasing $1 b_{1}{ }^{\prime}$ line width.

and if the line width would be allowed to change with temperature we can obtain an equal ratio between the two components as we enter into "no-man's land." Fig. 7 shows how we can fit the spectra with temperature-dependent line shapes to reconcile the expected nearly equal ratio between structures with tetrahedral and distorted H-bonds in terms of the area of the $1 b_{1}{ }^{\prime}$ and $1 b_{1}{ }^{\prime \prime}$ components at $222 \mathrm{~K}$. The basis for such a variation is a proposed picture of how the structure of the liquid evolves with decreasing temperature. ${ }^{18}$

In this picture, water behaves as a simple liquid at temperatures close to the boiling point, where most interactions are isotropic and dominated by non-directional van der Waals interactions. ${ }^{71}$ As the liquid cools down, the water molecules start to stick to each other through directional H-bonds. This will appear through two classes of configurations, ${ }^{18,24,28,29}$ tetrahedral structures with four strong H-bonds (LDL-like regions) and asymmetric structures with distorted H-bonds (HDL-like regions), with fluctuations between them on some timescale. The first class of structures is enthalpically favored due to efficient H-bonding. Since cooperativity effects make the bonds stronger if water is $\mathrm{H}$-bonded to other waters that are also in tetrahedral structures, fluctuations into tetrahedral structures expand into small local regions that become LDLlike in terms of their structure. The second class of structures is characterized by asymmetric local coordination with fewer, but paired, donor-acceptor H-bonds. With less directional $\mathrm{H}$ bonds there is more flexibility for librational and translational motions and thereby higher entropy. The $\mathrm{OH}$ groups that are involved in either a strong or weak H-bond can switch on

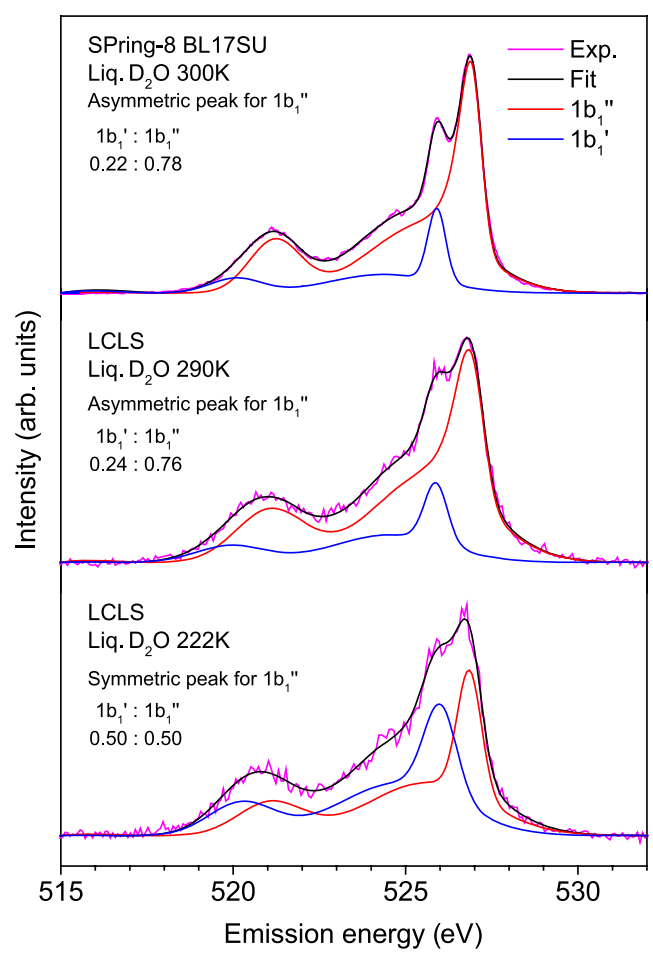

FIG. 7. Line-width dependent peak-fit analysis of XE spectra from SPring-8 at $300 \mathrm{~K}$ (top) and LCLS at $\sim 290 \mathrm{~K}$ (middle) with a dominating high-energy $1 b_{1}{ }^{\prime \prime}$ component and from LCLS at $222 \mathrm{~K}$ (bottom) with a close to $1: 1$ ratio between the $1 b_{1}{ }^{\prime}$ and $1 b_{1}{ }^{\prime \prime}$ components. The spectrum at $222 \mathrm{~K}$ has a broader $1 b_{1}{ }^{\prime}$ peak and a symmetric $1 b_{1}{ }^{\prime \prime}$ peak compared to the spectra close at higher temperature with narrower $1 b_{1}{ }^{\prime}$ peaks and asymmetric $1 b_{1}{ }^{\prime \prime}$ peaks.

a very fast timescale. In this class of structures, isotropic molecular van der Waals interactions are important causing interstitial molecules to come in from a collapsed 2nd shell also generating distortions around the 1st shell. Since in this case there are more water molecules in the first coordination shell (defined out to $3.5 \AA$ rather than to the minimum in $r^{2} g_{\text {OO }}$ (r) at $3.30 \AA^{72}$ ) than in the tetrahedral structures, we call these HDL-like structures.

There are fluctuations between the LDL-like and HDLlike structures. Although the timescale of these fluctuations slows down as we cool the liquid, the molecules in the asymmetric structures with distorted $\mathrm{H}$-bonds fluctuate more and more frequently into tetrahedral structures with four strong H-bonds, which grow in size since the balance between enthalpy and entropy in the free energy shifts more and more to favor enthalpy with decreasing temperature. There is also a continuous change in the structure of the HDLlike species with temperature. The switching time of $\mathrm{OH}$ groups participating in strong or weak/broken H-bonding is furthermore expected to slow down and the amplitude in the motion to decrease with temperature. The HDL-like regions will approach more and more tetrahedral character with strong $\mathrm{H}$-bonds as in high-density ices with many interstitials rather than as in hexagonal ice. Here, we propose that as the HDLlike structures become more symmetrical, similar to HDA ice, the two $\mathrm{OH}$ groups in the water molecule encounter more similar environments. This would lead to increased vibrational lifetime interference due to both $\mathrm{OH}$ groups contributing in the core-excited electronic state, rather than only one. This 
would make the line shape of the high-energy $1 b_{1}{ }^{\prime \prime}$ peak more symmetrical, similar to the $1 b_{1}{ }^{\prime}$ peak, and also narrower from the smaller thermal motion at lower temperature. As water is supercooled, the correlation length increases ${ }^{3}$ indicating that the LDL-like regions become significantly larger. We would therefore assume that there would be different subclasses of tetrahedral molecules depending on the long-range ordering, where some are in the center of the region and others at the boundary. This could lead to variations in the position of the $1 b_{1}$ emission energy and lead to a substantial broadening of the low-energy $1 b_{1}{ }^{\prime}$ peak, which would be fully consistent with the fitting shown in Fig. 7.

\section{SUMMARY}

Though all of the six hypotheses have different strengths and weaknesses, we regard the most likely explanation for the subtle changes in the lone-pair spectral region to be provided by the 6th hypothesis with minor contributions from the other mechanisms. If we assume a plausible lineshape temperature dependence, the ratio between $1 b_{1}{ }^{\prime}$ and $1 b_{1}{ }^{\prime \prime}$ becomes closer to what we expect, where the now smaller deviation from the expected behavior could come from beam-induced local structural transformations of tetrahedral to distorted $\mathrm{H}$-bonds. Clearly, this requires further investigation in the future utilizing both experiments and theory. Here, we would like to remark that the interpretation of XES of liquid water is indeed less straightforward than previously anticipated and other assumptions, such as temperaturedependent line shapes and potential beam-induced structural transformations of water, may be necessary to explain XE spectra of water upon deep supercooling. To further determine the line shape in the supercooled regime, it would be essential to measure water with superior energy resolution.

\section{ACKNOWLEDGMENTS}

We acknowledge the National Science Foundation (US) (Grant No. CHE-0809324), the Department of Energy through the SLAC Laboratory Directed Research and Development Program, Office of Basic Energy Sciences (BES) through SSRL and LCLS, the AMOS program within the Chemical Sciences, Geosciences, and Biosciences Division of the Office of BES, the Swedish Research Council, Lennanders Stiftelse, the Volkswagen Stiftung, and the Helmholtz Virtual Institute Dynamic Pathways in Multidimensional Landscapes for financial support. Portions of this research were carried out at the LCLS at the SLAC National Accelerator Laboratory. LCLS is an Office of Science User Facility operated for DOE Office of Science by Stanford University. Support from LCLS staff is gratefully acknowledged. The SXR instrument is funded by a consortium whose membership includes the LCLS, Stanford University through the Stanford Institute for Materials Energy Sciences (SIMES), Lawrence Berkeley National Laboratory (LBNL), University of Hamburg through the BMBF priority program FSP 301, and the Center for Free Electron Laser Science (CFEL). We thank Nils Mårtensson from Uppsala University and Franz Hennies from the MAX IV Laboratory for making available the XE spectrometer. The calculations were performed on resources provided by the Swedish National Infrastructure for Computing (SNIC) at the HPC2N center.

${ }^{1}$ H. Kanno and C. A. Angell, "Water: Anomalous compressibilities to $1.9 \mathrm{kbar}$ and correlation with supercooling limits," J. Chem. Phys. 70, 4008 (1979).

${ }^{2}$ R. J. Speedy and C. A. Angell, "Isothermal compressibility of supercooled water and evidence for a thermodynamic singularity at $-45^{\circ} \mathrm{C}$," J. Chem. Phys. 65, 851 (1976).

${ }^{3} \mathrm{C}$. Huang et al., "Increasing correlation length in bulk supercooled $\mathrm{H}_{2} \mathrm{O}$, $\mathrm{D}_{2} \mathrm{O}$, and $\mathrm{NaCl}$ solution determined from small angle x-ray scattering," $\mathrm{J}$. Chem. Phys. 133, 134504 (2010).

${ }^{4}$ B. J. Mason, "The supercooling and nucleation of water," Adv. Phys. 7, 221-234 (1958).

${ }^{5}$ P. H. Poole, F. Sciortino, U. Essmann, and H. E. Stanley, "Phase-behavior of metastable water," Nature 360, 324-328 (1992).

${ }^{6}$ S. Sastry, P. G. Debenedetti, F. Sciortino, and H. E. Stanley, "Singularityfree interpretation of the thermodynamics of supercooled water," Phys. Rev. E 53, 6144-6154 (1996).

${ }^{7}$ C. A. Angell, "Insights into phases of liquid water from study of its unusual glass-forming properties," Science 319, 582 (2008).

${ }^{8} \mathrm{D}$. T. Limmer and D. Chandler, "The putative liquid-liquid transition is a liquid-solid transition in atomistic models of water," J. Chem. Phys. 135, 134503 (2011).

${ }^{9} \mathrm{P}$. Gallo and F. Sciortino, "Ising universality class for the liquid-liquid critical point of a one component fluid: A finite-size scaling test," Phys. Rev. Lett. 106, 177801 (2012).

${ }^{10}$ T. A. Kesselring, G. Franzese, S. V. Buldyrev, H. J. Herrmann, and H. E. Stanley, "Nanoscale dynamics of phase flipping in water near its hypothesized liquid-liquid critical point," Sci. Rep. 2, 474 (2012).

${ }^{11}$ T. A. Kesselring, E. Lascaris, G. Franzese, and H. E. Stanley, "Finite-size scaling investigation of the liquid-liquid critical point in ST2 water and its stability with respect to crystallization," J. Chem. Phys. 138, 244506 (2013).

${ }^{12}$ D. T. Limmer and D. Chandler, "The putative liquid-liquid transition is a liquid-solid transition in atomistic models of water. II," J. Chem. Phys. 138, 214504 (2013).

${ }^{13}$ J. C. Palmer, R. Car, and P. G. Debenedetti, "The liquid-liquid transition in supercooled ST2 water: A comparison between umbrella sampling and well-tempered dynamics," Faraday Discuss. 167, 77-94 (2013).

${ }^{14} \mathrm{~J}$. C. Palmer et al., "Metastable-liquid-liquid transition in a molecular model of water," Nature 510, 385-388 (2014).

${ }^{15}$ R. J. Speedy, "Stability-limit conjecture. An interpretation of the properties of water," J. Phys. Chem. 86, 982 (1982).

${ }^{16} \mathrm{E}$. B. Moore and V. Molinero, "Structural transformation in supercooled water controls the crystallization rate of ice," Nature 479, 506-508 (2011).

${ }^{17} \mathrm{~J}$. A. Sellberg et al., "Ultrafast X-ray probing of water structure below the homogeneous ice nucleation temperature," Nature 510, 381-384 (2014).

${ }^{18}$ A. Nilsson and L. G. M. Pettersson, "Perspective on the structure of liquid water," Chem. Phys. 389, 1-34 (2011).

${ }^{19} \mathrm{O}$. Fuchs et al., "Isotope and temperature effects in liquid water probed by x-ray absorption and resonant x-ray emission spectroscopy," Phys. Rev. Lett. 100, 027801 (2008).

${ }^{20} \mathrm{~T}$. Tokushima et al., "High resolution x-ray emission spectroscopy of liquid water: The observation of two structural motifs," Chem. Phys. Lett. 460, $387-400$ (2008).

${ }^{21} \mathrm{~K}$. M. Lange et al., "High resolution x-ray emission spectroscopy of water and aqueous ions using the micro-jet technique," Chem. Phys. 377, 1-5 (2010).

${ }^{22} \mathrm{~K}$. M. Lange et al., "On the origin of the hydrogen-bond-network nature of water: X-ray absorption and emission spectra of water-acetonitrile mixtures," Angew. Chem. 123, 1-6 (2011).

${ }^{23} \mathrm{~K}$. M. Lange et al., "X-ray emission from pure and dilute $\mathrm{H} 2 \mathrm{O}$ and $\mathrm{D} 2 \mathrm{O}$ in a liquid microjet: Hydrogen bonds and nuclear dynamics," Phys. Rev. B 85, 155104 (2012).

${ }^{24}$ A. Nilsson, C. Huang, and L. G. M. Pettersson, "Fluctuations in ambient water," J. Mol. Liq. 176, 2-16 (2012).

${ }^{25} \mathrm{~A}$. Nilsson et al., "Resonant inelastic x-ray scattering of liquid water," J. El. Struc. Rel. Phenom. 188, 84-100 (2013).

${ }^{26} \mathrm{~T}$. Tokushima et al., "High resolution x-ray emission spectroscopy of water and its assignment based on two structural motifs," J. Electron Spectrosc. Relat. Phenom. 177, 192-205 (2010).

${ }^{27} \mathrm{~T}$. Tokushima et al., "Polarization dependent resonant $\mathrm{x}$-ray emission spectroscopy of $\mathrm{D}_{2} \mathrm{O}$ and $\mathrm{H}_{2} \mathrm{O}$ water: Assignment of the local molecular orbital symmetry," J. Chem. Phys. 136, 044517 (2012). 
${ }^{28} \mathrm{C}$. Huang et al., "The inhomogeneous structure of water at ambient conditions," Proc. Natl. Acad. Sci. U. S. A. 106, 15214-15218 (2009).

${ }^{29}$ L. G. M. Pettersson and A. Nilsson, "The structure of water; from ambient to deeply supercooled," J. Non-Cryst. Solids 407, 399-417 (2015).

${ }^{30} \mathrm{O}$. Fuchs et al., "Response to "Comment on 'Isotope and temperature effects in liquid water probed by $\mathrm{x}$-ray absorption and resonant $\mathrm{x}$-ray emission spectroscopy,"', Phys. Rev. Lett. 100, 249802 (2008).

${ }^{31} \mathrm{M}$. Odelius, "Information content in O[1s] k-edge $\mathrm{x}$-ray emission spectroscopy of liquid water," J. Phys. Chem. A 113, 8176-8181 (2009).

${ }^{32} \mathrm{M}$. Odelius, "Molecular dynamics simulations of fine structure in oxygen $\mathrm{k}$ edge x-ray emission spectra of liquid water and ice," Phys. Rev. B 79, 144204 (2009).

${ }^{33}$ J. Forsberg et al., "Angular anisotropy of resonant inelastic soft x-ray scattering from liquid water," Phys. Rev. B 79, 132203 (2009).

${ }^{34}$ M. P. Ljungberg, A. Nilsson, and L. G. M. Pettersson, "Semi-classical description of nuclear dynamics in x-ray emission of water," Phys. Rev. B 82, 245115 (2010).

${ }^{35}$ M. P. Ljungberg, L. G. M. Pettersson, and A. Nilsson, "Vibrational interference effects in X-ray emission of a model water dimer: Implications for the interpretation of the liquid spectrum," J. Chem. Phys. 134, 044513 (2011).

${ }^{36} \mathrm{~W}$. F. Schlotter et al., "The soft x-ray instrument for materials studies at the linac coherent light source x-ray free-electron laser," Rev. Sci. Instrum. 83, 043107 (2012)

${ }^{37}$ S. Schreck et al., "Reabsorption of soft x-ray emission at high x-ray freeelectron laser fluences," Phys. Rev. Lett. 113, 153002 (2014).

${ }^{38}$ J. Nordgren et al., "X-ray spectrometer," Rev. Sci. Instrum. 60, 1690 (1989).

${ }^{39} \mathrm{~K}$. Kunnus et al., "A setup for resonant inelastic soft X-ray scattering on liquids at free electron laser light sources," Rev. Sci. Instrum. 83, 123109 (2012).

${ }^{40}$ D. P. DePonte et al., "Gas dynamic virtual nozzle for generation of microscopic droplet streams," J. Phys. D: Appl. Phys. 41, 195505 (2008).

${ }^{41}$ F. R. S. Rayleigh, "On the instability of jets," Proc. London Math. Soc. s1-10, 4-13 (1878).

${ }^{42}$ M. Knudsen, "Die maximale verdampfungsgeschwindigkeit des quecksilbers," Ann. Phys. 352, 697-708 (1915).

${ }^{43}$ J. R. Maa, "Evaporation coefficient of liquids," Ind. Eng. Chem. Fundam. 6, 504-518 (1967).

${ }^{44}$ J. D. Smith, C. D. Cappa, W. S. Drisdell, R. C. Cohen, and R. J. Saykally, "Raman thermometry measurements of free evaporation from liquid water droplets," J. Am. Chem. Soc. 128, 12892-12898 (2006).

${ }^{45}$ T. Tokushima, Y. Harada, H. Ohashi, Y. Senba, and S. Shin, "High performance slit-less spectrometer for soft X-ray emission spectroscopy," Rev. Sci. Instrum. 77, 063107 (2006).

${ }^{46}$ Y. Harada et al., "Ultrahigh resolution soft X-ray emission spectrometer at BL07LSU in SPring-8," Rev. Sci. Instrum. 83, 013116 (2012).

${ }^{47}$ V. N. Strocov, T. Schmitt, U. Flechsig, L. Patthey, and G. S. Chiuzbăian, "Numerical optimization of spherical variable-line-spacing grating x-ray spectrometers," J. Synchrotron Radiat. 18, 134-142 (2011).

${ }^{48}$ M. Ogawa et al., "Development of soft x-ray time-resolved photoemission spectroscopy system with a two-dimensional angle-resolved time-of-flight analyzer at SPring-8 BL07LSU,” Rev. Sci. Instrum. 83, 023109 (2012).

${ }^{49} \mathrm{~A}$. Møgelhøj et al., "Ab initio van der Waals interactions in simulations of water alter structure from mainly tetrahedral to high-density-like," J. Phys. Chem. B 115, 14149-14160 (2011).

${ }^{50}$ A. Föhlisch et al., "Ground state interpretation of XES of adsorbates," Phys. Rev. B 61, 16229 (2000).

${ }^{51}$ A. M. Köster et al., deMon 2k, version 3, deMon Developers, Mexico City, 2011.
${ }^{52}$ B. Hammer, L. B. Hansen, and J. K. Nørskov, "Exchange functional rPBE," Phys. Rev. B 59, 7413 (1999).

${ }^{53} \mathrm{~W}$. Kutzelnigg, U. Fleischer, and M. Schindler, NMR-Basic Principles and Progress (Springer Verlag, Heidelberg, 1990).

${ }^{54}$ G. Igel-Mann, H. Stoll, and H. Preuss, "Pseudopotentials for main group elements (IIIa through VIIa)," Mol. Phys. 65, 1321-1328 (1988).

${ }^{55}$ E. Gilberg, M. J. Hanus, and B. Foltz, "Investigation of the electronicstructure of ice by high-resolution x-ray spectroscopy," J. Chem. Phys. 76, 5093-5097 (1982).

${ }^{56}$ K. T. Wikfeldt, A. Nilsson, and L. G. M. Pettersson, "Spatially inhomogeneous bimodal inherent structure in simulated liquid water," Phys. Chem. Chem. Phys. 13, 19918-19924 (2011).

${ }^{57} \mathrm{D}$. Nordlund et al., "The electronic structure of liquid water studied using photoelectron spectroscopy," Chem. Phys. Lett. 460, 86 (2008).

${ }^{58} \mathrm{~B}$. Winter et al., "Full valence band photoemission from liquid water using EUV synchrotron radiation,” J. Phys. Chem. A 108, 2625 (2004).

${ }^{59}$ K. Nishizawa, N. Kurahashi, K. Sekiguchi, and T. Mizuno, "High-resolution soft x-ray photoelectron spectroscopy of liquid water," Phys. Chem. Chem. Phys. 13, 413-417 (2011).

${ }^{60}$ B. Winter, E. F. Aziz, U. Hergenhahn, M. Faubel, and I. V. Hertel, "Hydrogen bonds in liquid water studied by photoelectron spectroscopy," J. Chem. Phys. 126, 124504 (2007).

${ }^{61} \mathrm{~A}$. Nilsson et al., "The hydrogen bond in ice probed by soft x-ray spectroscopy and density functional theory," J. Chem. Phys. 122, 154505 (2005).

${ }^{62} \mathrm{~S}$. Thürmer et al., "On the nature and origin of dicationic, charge-separated species formed in liquid water on x-ray irradiation," Nat. Chem. 5, 590-596 (2013).

${ }^{63} \mathrm{~J}$. A. Sellberg et al., "Comparison of x-ray absorption spectra between water and ice: New ice data with low pre-edge absorption cross-section," J. Chem. Phys. 141, 034507 (2014).

${ }^{64}$ O. Mishima, L. D. Calvert, and E. Whalley, “"Melting ice' I at $77 \mathrm{~K}$ and 10 kbar: A new method of making amorphous solids," Nature 310, 393-395 (1984).

${ }^{65}$ E. T. J. Nibbering and T. Elsaesser, "Ultrafast vibrational dynamics of hydrogen bonds in the condensed phase," Chem. Rev. 104, 1887-1914 (2004).

${ }^{66}$ K. T. Wikfeldt, C. Huang, A. Nilsson, and L. G. M. Pettersson, "Enhanced small-angle scattering connected to the Widom line in simulations of supercooled water," J. Chem. Phys. 134, 214506 (2011).

${ }^{67} \mathrm{~J}$. E. Bertie and Z. Lan, "Infrared intensities of liquids XX: The intensity of the $\mathrm{OH}$ stretching band of liquid water revisited, and the best current values of the optical constants of $\mathrm{H} 2 \mathrm{O}(\mathrm{l})$ at $25 \infty \mathrm{C}$ between 15000 and $1 \mathrm{~cm}^{-1}$," Appl. Spectrosc. 50, 1047-1057 (1996).

${ }^{68}$ C. J. Fecko, J. D. Eaves, J. J. Loparo, A. Tokmakoff, and P. L. Geissler, "Ultrafast hydrogen-bond dynamics in the infrared spectroscopy of water," Science 301, 1698-1702 (2003).

${ }^{69}$ A. K. Soper and M. A. Ricci, "Structures of high-density and low-density water," Phys. Rev. Lett. 84, 2881-2884 (2000).

${ }^{70} \mathrm{~A}$. Nilsson et al., "X-ray absorption spectroscopy and x-ray Raman scattering of water; an experimental view," J. Electron Spectrosc. Relat. Phenom. 177, 99-129 (2010).

${ }^{71}$ S. V. Lishchuk, N. P. Malomuzh, and P. V. Makhlaichuk, "Why thermodynamic properties of normal and heavy water are similar to those of argon-like liquids?,” Phys. Lett. A 374, 2084 (2010).

${ }^{72}$ L. B. Skinner et al., "Benchmark oxygen-oxygen pair-distribution function of ambient water from x-ray diffraction measurements with a wide Q-range," J. Chem. Phys. 138, 074506 (2013). 